\title{
Toll-like receptor-induced changes in glycolytic metabolism regulate dendritic cell activation
}

\author{
Connie M. Krawczyk, ${ }^{1}$ Thomas Holowka, ${ }^{1}$ Jie Sun, ${ }^{1}$ Julianna Blagih, $, 2,3$ Eyal Amiel, ${ }^{4}$ Ralph J. DeBerardinis, ${ }^{5}$ Justin R. Cross, ${ }^{6}$ \\ Euihye Jung, ${ }^{1}$ Craig B. Thompson, ${ }^{6-8}$ Russell G. Jones, ${ }^{2,3}$ and Edward J. Pearce ${ }^{1,4}$ \\ ${ }^{1}$ Department of Pathobiology, School of Veterinary Medicine, University of Pennsylvania, Philadelphia; ${ }^{2}$ Goodman Cancer Research Centre and ${ }^{3}$ Department of \\ Physiology, McGill University, Montreal, QC; ${ }^{4}$ Trudeau Institute, Saranac Lake, NY; ${ }^{5}$ Department of Pediatrics and Genetics, University of Texas Southwestern \\ Medical Center, Dallas; and ${ }^{6}$ Abramson Family Cancer Research Institute, ${ }^{7}$ Department of Cancer Biology, and ${ }^{8}$ Faculty of Medicine, University of Pennsylvania, \\ Philadelphia
}

\begin{abstract}
Dendritic cells (DCs) are key regulators of innate and acquired immunity. The maturation of DCs is directed by signal transduction events downstream of toll-like receptors (TLRs) and other pattern recognition receptors. Here, we demonstrate that, in mouse DCs, TLR agonists stimulate a profound metabolic transition to aerobic glycolysis, similar to the Warburg
\end{abstract}

metabolism displayed by cancer cells. This metabolic switch depends on the phosphatidyl inositol 3'-kinase/Akt pathway, is antagonized by the adenosine monophosphate (AMP)-activated protein kinase (AMPK), and is required for DC maturation. The metabolic switch induced by DC activation is antagonized by the antiinflammatory cytokine interleukin-10.
Our data pinpoint TLR-mediated metabolic conversion as essential for DC maturation and function and reveal it as a potential target for intervention in the control of excessive inflammation and inappropriately regulated immune responses. (Blood. 2010;115(23):4742-4749)

\section{Introduction}

Toll-like receptor (TLR)-directed dendritic cell (DC) activation is critical for the tailoring of immune responses to specific pathogens. ${ }^{1,2}$ TLR agonists initiate profound changes in DCs, which without undergoing cellular division transition from a resting state into an activated mature state. ${ }^{3,4}$ This process involves large-scale changes in the expression of genes encoding a broad array of proteins, including but not limited to cytokines, chemokines, and costimulatory molecules, ${ }^{5}$ and behavioral changes that result in DCs migrating into T-cell areas within lymphoid organs. ${ }^{6}$

We hypothesized that, like growth factors, TLR agonists might not only direct new gene transcription but also affect changes in cellular metabolic pathways to ensure that sufficient bioenergetic and metabolic resources exist to support programs of new gene expression. ${ }^{7,8}$ Consistent with this, signaling pathways elicited by TLRs share elements with those used by growth factor receptors. For example, TLRs have been shown to initiate phosphatidyl inositol 3'-kinase (PI3K)/Akt activation in a variety of cell types, including DCs, ${ }^{9,10}$ although the full consequences of this remain unclear. Moreover, in macrophages TLR ligation initiates autophagy, ${ }^{11,12}$ a process that contributes to the killing of pathogens within cells, but also provides metabolic intermediates to maintain bioenergetics and promote cell survival.

Here, we show that after exposure to TLR agonists, DCs undergo a metabolic transition from oxidative phosphorylation (OXPHOS) to aerobic glycolysis. This process underpins the activation pathway, because its inhibition results in a failure of DCs to mature. The metabolic switch is promoted by PI3K/Akt signaling and is inhibited by the adenosine monophosphate (AMP)activated protein kinase (AMPK), a central regulator of catabolic metabolism and OXPHOS, ${ }^{13}$ and by the antiinflammatory cytokine interleukin-10 (IL-10). ${ }^{14}$ Collectively, the data implicate DC metabolic reprogramming as an underlying control point for the inflammatory processes and adaptive immune responses induced by this important cell type.

\section{Methods}

\section{Mice and reagents}

C57BL6 (B6) mice were purchased from The Jackson Laboratory, and $a k t 1^{-/-}$mice (a gift from Dr M. Birnbaum, University of Pennsylvania) and DO11.10 mice were bred and maintained under specific pathogen-free conditions at the University of Pennsylvania under protocols approved by the Institutional Animal Care and Use Committee. Heat-killed Propionibacterium acnes (PA) was purchased from The Van Kampen Group. Cytosine phosphate guanine oligodeoxynucleotide 1826 was obtained from Coley Pharmaceuticals. Lipopolysaccharide (LPS; Escherichia coli serotype 0111:B4) was from Sigma-Aldrich. Inhibitors and activators were from Sigma-Aldrich. Golgi-Plug (brefeldin) and antibodies for flow cytometry, enzyme-linked immunoabsorbent assays, and against IL-10 receptor (IL10R) were from BD PharMingen. MitoTrackers were from Invitrogen. Total and phosphospecific Akt and AMPK antibodies were from Cell Signaling Technologies. All media components were from Mediatech.

\section{DC culture and retroviral transduction}

Bone marrow-derived DCs were generated as described. ${ }^{15}$ Briefly, bone marrow DC precursors were differentiated in the presence of granulocytemacrophage colony-stimulating factor (GM-CSF; $20 \mathrm{ng} / \mathrm{mL}$ ) in complete DC media (RPMI containing 10\% fetal calf serum, $100 \mathrm{U} / \mathrm{mL}$ penicillin/
Submitted October 22, 2009; accepted February 28, 2010. Prepublished online as Blood First Edition paper, March 29, 2010; DOI 10.1182/blood-2009-10-249540.

The online version of this article contains a data supplement.
The publication costs of this article were defrayed in part by page charge payment. Therefore, and solely to indicate this fact, this article is hereby marked "advertisement" in accordance with 18 USC section 1734. 
streptomycin, $0.05 \mathrm{mM}$ basal medium Eagle, and 2mM L-glutamine). Retroviral transduction of DCs was accomplished as described previously. ${ }^{16}$ Sequences for AMPK short hairpin RNAs (shRNAs) were obtained from Open Biosystems and cloned into LMP retroviral vectors; the shRNAs were highly effective as judged by their ability to knockdown AMPK levels in mouse embryonic fibroblasts (supplemental Figure 6, available on the Blood Web site; see the Supplemental Materials link at the top of the online article). Empty (vector) or recombinant retroviruses were obtained after the transfection of 293T packaging cells with the use of Lipofectamine (Invitrogen); retrovirus-containing supernatants were collected 48 hours after transfection and used for spin infection (2500 rpm, 2 hours) of day 1 bone marrow DC cultures in 6-well plates. After an additional 7 to 8 days in culture with GM-CSF, DCs were harvested, and transduction efficiency was assessed with the use of anti-human CD8 antibody (Ab) and flow cytometry; we typically obtained transduction rates greater than $80 \%$. On days 8 to 10 of culture, DCs were washed into complete DC media containing $5 \mathrm{ng} / \mathrm{mL}$ GM-CSF and various concentrations of glucose or 2-deoxyglucose and were maintained in medium alone or pulsed for 18 hours with heat-killed PA $(10 \mu \mathrm{g} / \mathrm{mL})$, cytosine phosphate guanine oligodeoxynucleotide $1826(1 \mu \mathrm{g} / \mathrm{mL})$, or LPS $(100 \mathrm{ng} / \mathrm{mL})$. In some experiments IL-10 (50 ng/mL), monoclonal (mAb) anti-IL-10R $(30 \mu \mathrm{g} /$

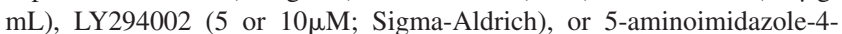
carboxamide ribonucleotide (AICAR; $1 \mathrm{mM}$ ) were added to DC cultures.

\section{Western blotting}

Cell lysate preparation, sodium dodecyl sulfate-polyacrylamide gel electrophoresis, electrophoretic transfer, immunoblotting, and development with enhanced chemiluminescence were accomplished as described previously. ${ }^{17}$

\section{Cytokine measurements and flow cytometry}

Intracellular cytokine staining and staining for costimulatory molecules and major histocompatibility complex II were performed with specific fluorochrome-labeled mAbs and flow cytometry, as described. ${ }^{2}$ Mitochondrial mass and potential were measured with the use of MitoTracker Green FM and MitoTracker Red CMXRos, respectively, and flow cytometry according to the manufacturer's instructions. Cytokines in DC supernatants were measured by capture enzyme-linked immunoabsorbent assays with the use of paired mAbs and recombinant standards, as described. ${ }^{18}$

\section{In vivo T-cell responses}

Mice were adoptively transferred with $5 \times 10^{6}$ 5,6-carboxyfluorescein diacetate, succinimidyl ester (CFSE)-labeled DO11.10 splenocytes intravenously and 1 day later injected subcutaneously into one rear footpad with $5 \times 10^{5}$ washed DCs that had previously been cultured in medium alone, ovalbumin (OVA) alone (300 $\mu \mathrm{g} / \mathrm{mL}$ endotoxin-free egg white), OVA plus LPS $(100 \mathrm{ng} / \mathrm{mL})$, or OVA plus LPS in the presence of 2-deoxyglucose (2-DG; $10 \mathrm{mM}$ ) for 6 hours. Four days later, mice were killed, and popliteal lymph nodes (LNs) were harvested. Single-cell suspensions were stained with fluorochrome-conjugated $\mathrm{mAb}$ specific for the DO11.10 clonotype (KJ126) and CD4 and were analyzed by flow cytometry. In some experiments, mice were injected subcutaneously into one rear footpad with $5 \times 10^{5}$ washed, CFSE-labeled DCs that had previously been treated as described in this section. Two days later draining popliteal LNs were isolated, and CD11 $\mathrm{c}^{+}$CFSE-labeled cells were analyzed by flow cytometry.

\section{Real-time RT-PCR}

DCs were pulsed with different stimuli, as described in "In vivo T-cell responses," for 6 hours before RNA extraction. RNA was extracted with RNeasy (QIAGEN) and treated with DNase I (Invitrogen). Oligo (dT) (Promega) and Superscript II (Invitrogen) were used to synthesize first-strand cDNAs from equivalent amounts of RNA from each sample. Real-time reverse transcriptionpolymerase chain reaction (RT-PCR) was performed in a 7500 Fast Real-Time PCR System (Applied Biosystems) with SYBR green RT-PCR master mix (Applied Biosystems). Data were generated with the comparative threshold cycle $\left(\delta \mathrm{C}_{\mathrm{T}}\right)$ method by normalizing to hypoxanthine phospho ribosyl transferase. Sequences of primers used in the studies are available on request.

\section{Metabolic assays}

The glycolytic rate of DCs was determined by measuring the conversion of $5-{ }^{3} \mathrm{H}$-glucose to tritiated water, as described previously. ${ }^{19}$ Immature DCs were plated at $1 \times 10^{6} / \mathrm{mL}$ in 24-well plates and left unstimulated or stimulated with various TLR ligands for 14 to 16 hours. Cells were harvested, washed twice, and resuspended in glucose-free medium (RPMI; Gibco), and then incubated with $10 \mu \mathrm{Ci}(0.037 \mathrm{~Bq})$ of $5-{ }^{3} \mathrm{H}$-glucose (Perkin-Elmer) at $37^{\circ} \mathrm{C}$ for 1 hour. The reaction was stopped by adding $\mathrm{HCl}$ (0.1M final). $\left[{ }^{3} \mathrm{H}\right]_{2} \mathrm{O}$ generated by enolase activity was separated from $5-{ }^{3} \mathrm{H}$-glucose by diffusion, and counts were measured with the use of a 1450 Microbeta scintillation counter (Wallac). The glycolytic rate for each sample was normalized relative to that of unstimulated, immature DCs within each experiment and expressed as fold increase. Glucose and lactate levels in culture medium were measured with the use of a BioProfile FLEX analyzer (NOVA Biomedical).

Measurement of mitochondrial-dependent $\beta$-oxidation of fatty acids was conducted as previously described with modifications. ${ }^{20}$ In brief, $1 \times 10^{6}$ DCs were left unstimulated or stimulated with $100 \mathrm{ng} / \mathrm{mL}$ LPS for 14 hours. $\left[9,10-{ }^{3} \mathrm{H}\right]$-palmitic acid complexed to bovine serum albumin (essentially fatty acid free; Sigma-Aldrich) was added to the DC cultures and incubated for 6 hours. Mitochondrial-independent $\beta$-oxidation was assessed by measuring $\left[9,10-{ }^{3} \mathrm{H}\right]$-palmitate oxidation in the presence of $200 \mu \mathrm{M}$ etomoxir (Sigma-Aldrich), an irreversible inhibitor of carnitine palmitoyltransferase I, the rate-limiting enzyme for mitochondrial import of long-chain fatty acids. Supernatant was applied to ion-exchange columns (Dowex $1 \times 8$-200; Sigma-Aldrich), and tritiated water was recovered by eluting the columns with water. One-fifth of the recovered tritiated water was then used for scintillation counting. The rate of $\beta$-oxidation was calculated as the difference between oxidation counts in the presence or absence of etomoxir and was expressed as count per minute per $1 \times 10^{6}$ cells.

The rate of oxygen consumption by LPS-activated DCs was determined as previously described with the use of a water-jacketed, air-tight oximeter (Hansatech). ${ }^{21}$ OXPHOS-dependent oxygen consumption was calculated as the difference between the total rate of oxygen consumption and the rate in the presence of $5 \mu \mathrm{M}$ oligomycin, an inhibitor of mitochondrial adenosine triphosphate (ATP) synthase.

\section{Statistics}

Statistical significance was calculated with the Student $t$ test.

\section{Results}

\section{DC activation by TLR ligands induces a metabolic switch to glycolytic metabolism}

To determine whether TLR activation has effects on glycolytic metabolism, DCs were activated by various TLR ligands and analyzed for their ability to metabolize glucose, the primary substrate for ATP generation through glycolysis. Stimulation of DCs by TLR4, TLR2, and TLR9 promoted a significant increase in the glycolytic rate (2- to 4-fold) relative to resting controls (Figure 1A). Increased glycolysis requires greater usage of glucose, and in other cells this is facilitated by the increased expression of glucose transporters at the plasma membrane. ${ }^{22}$ Consistent with this, increased levels of glucose transporter 1 transcripts (Figure 1B) and protein (supplemental Figure 1) were observed after TLR stimulation of DCs. Both glucose consumption and the production of lactate, a metabolic end point of aerobic glycolysis, increased after LPS stimulation of DCs (Figure 1C-D), indicating an increase in glycolytic activity after TLR stimulation.

The up-regulation of glycolysis after DC activation prompted us to examine other metabolic pathways affected by TLR stimulation. Fatty acid $\beta$-oxidation by mitochondria is an important catabolic 

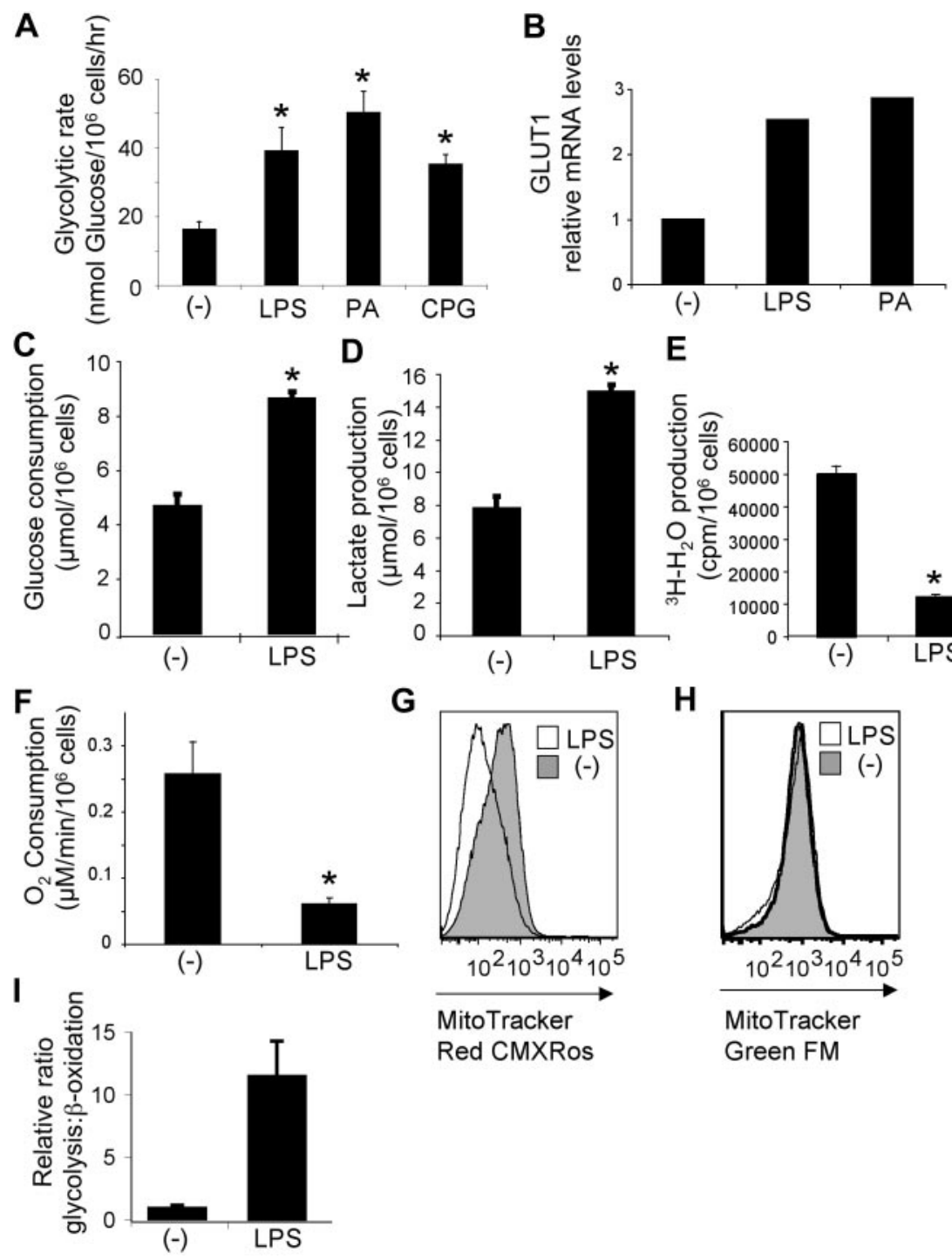

G

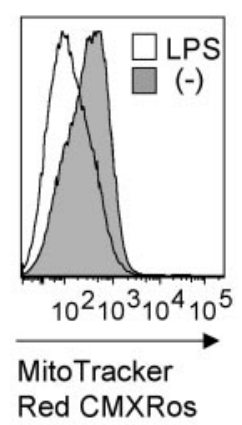

E

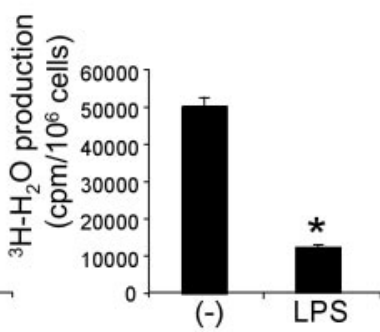

H

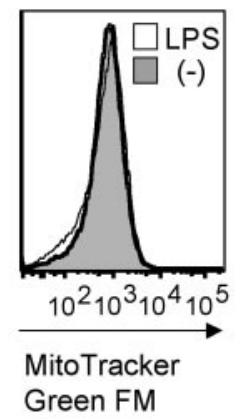

Figure 1. DC activation by TLR ligands induces a metabolic switch to aerobic glycolysis. (A) Glycolytic rate of DCs after18 hours of culture in medium alone (-) or with the TLR4, TLR2, and TLR9 ligands, LPS, heat-killed PA or cytosine phosphate guanosine (CpG). (B) Glucose transporter 1 (GLUT1) expression by DCs analyzed by quantitative RT-PCR. (C) Glucose consumption by resting (-) and LPS-activated DCs. (D) Lactate production by resting (-) and LPS-activated DCs. (E) Rate of mitochondrial fatty acid $\beta$-oxidation in DCs cultured for 18 hours in medium alone (-) or after stimulation with LPS. (F) Mitochondrial $\mathrm{O}_{2}$ consumption of resting (-) or LPSstimulated DCs. (G) Mitochondrial potential, as assessed by fluorescence with the MitoTracker Red CMXRos. (H) Mitochondrial mass, assessed by fluorescence with the MitoTracker Green FM. $(\mathrm{G}-\mathrm{H})$ The $y$-axis indicates cell number. (I) The glycolysis/ $\beta$-oxidation ratio for DCs stimulated with LPS. Bars represent mean values \pm SDs from 3 independent replicates; ${ }^{\star} P<.05$ compared with unstimulated controls (-). All data are representative of 2 to 4 independent experiments.

energy-generating pathway used for homeostatic processes in unstressed and resting cells. ${ }^{20} \mathrm{We}$ found that the rate of mitochondrial-dependent $\beta$-oxidation of free fatty acids was significantly suppressed after TLR activation (Figure 1E). Although overall oxygen consumption by DCs did not dramatically change after LPS stimulation, lower rates of mitochondrial-dependent oxygen consumption were observed (Figure 1F). Consistent with this reduction in mitochondrial oxygen consumption, mitochondrial potential also decreased after exposure to LPS (Figure 1G). This occurred in the absence of any change in mitochondrial mass (Figure $1 \mathrm{H}$ ). Taken together, these data indicate that TLR agonists promote a profound metabolic switch in resting immature DCs from mitochondrial $\beta$-oxidation and OXPHOS to aerobic glycolysis (Figure 1I). This switch to glycolysis despite ample oxygen to support OXPHOS is reminiscent of the metabolic switch induced on transformation (the "Warburg effect").

\section{PI3K/Akt signaling controls the metabolic switch to aerobic glycolysis}

The induction of glycolysis by growth factor receptors depends on signal transduction mediated by the PI3K/Akt pathway. ${ }^{22,23}$ The PI3K/Akt pathway was examined directly for its role in TLRinduced glycolysis in DCs. LPS-induced glycolysis was signifi- cantly reduced in DCs treated with the PI3K inhibitor LY294002 (Figure 2A) and in DCs lacking Akt1 (Figure 2B). In contrast, DCs retrovirally transduced to express an active form of $\mathrm{Akt}^{24}$ exhibited increased background levels of glycolysis and a heightened glycolytic response to LPS (Figure 2C). The PI3K/Akt pathway was also examined for its role in regulating the TLR-induced suppression of mitochondrial-dependent $\beta$-oxidation of fatty acids. Interestingly, the suppression of mitochondrial fatty acid $\beta$-oxidation triggered by LPS was unaffected by the inhibition of PI3K by LY294002 (Figure 2D), the absence of Akt1 (Figure 2E), or the expression of the active form of Akt (Figure 2F). These findings show that the PI3K/Akt pathway plays a pivotal role in regulating glycolytic metabolism in TLR-stimulated DCs but is dispensable for the accompanying down-regulation of mitochondrial $\beta$-oxidation.

\section{Glycolysis is essential for cellular activation and survival after exposure to TLR agonists}

The engagement of aerobic glycolysis by growth factor signaling allows cells to meet the bioenergetic demands of cellular proliferation. ${ }^{25}$ Although TLR signaling, like growth factor signaling, leads to increases in glycolytic metabolism, it does not promote DC proliferation. This implies that the metabolic changes, and specifically the increase in glycolysis triggered by TLRs, serve to meet the 
Figure 2. TLRs induce glycolytic metabolism in DCs through the PI3K/Akt pathway. (A) Glycolytic rate of DCs stimulated with LPS in the presence of the indicated concentrations of the PI3K inhibitor LY294002. (B) Glycolytic rate after LPS stimulation in $a k t 1^{+/+}$and $a k t 1^{-1}$ DCs. The fold change in glycolytic rate was calculated relative to the untreated control for each genotype. (C) Glycolytic rate of DCs transduced with the retroviral vectors MIG (control) or MIG-active form of Akt (myrAkt). The data represent the fold increase in glycolytic rate relative to unstimulated MIG-transduced DCs. (D) Rate of mitochondrial fatty acid $\beta$-oxidation in DCs cultured for 18 hours in medium alone (-) or after stimulation with LPS in the presence or absence of the PI3K inhibitor LY294002. (E) Rate of mitochondrial fatty acid $\beta$-oxidation in wild-type $\left(a k t 1^{+/+}\right)$or Akt-deficient $\left(a k t 1^{-/-}\right)$DCs cultured for 18 hours in medium alone $(-)$ or after stimulation with LPS. (F) Rate of mitochondrial fatty acid $\beta$-oxidation in DCs transduced with empty vector (MIG) or expressing myr-Akt. Bars represent mean value of replicates. Error bars represent SDs. All data are representative of 2 or more independent experiments.
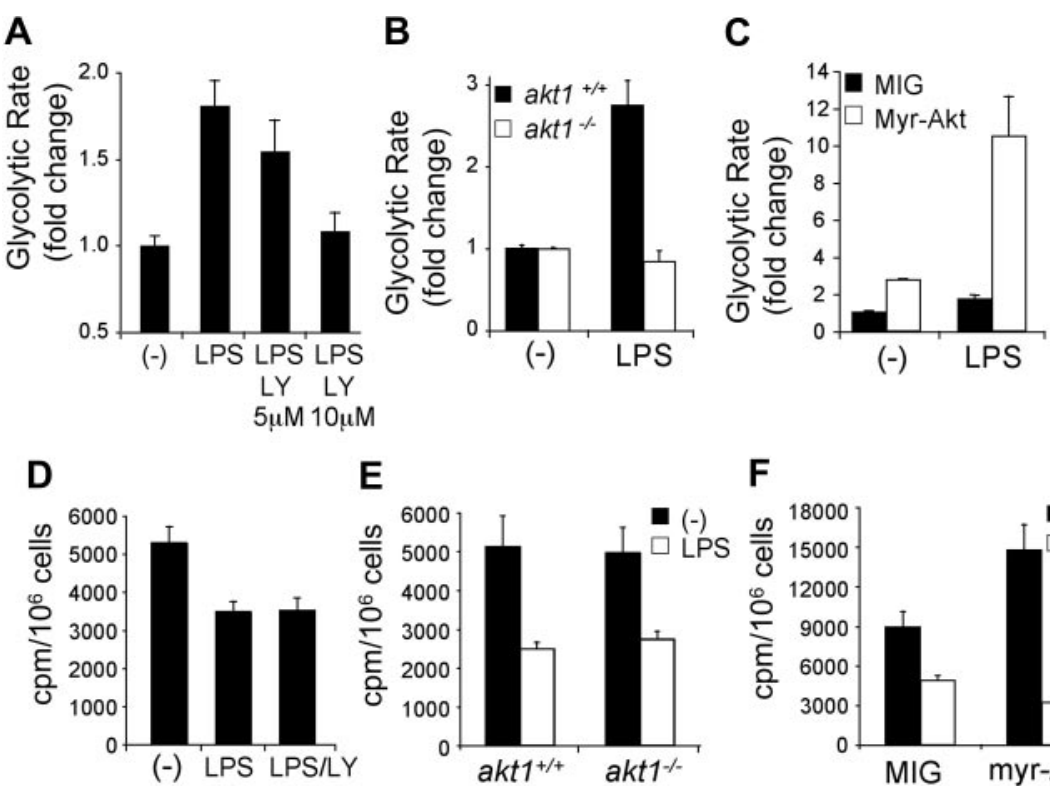

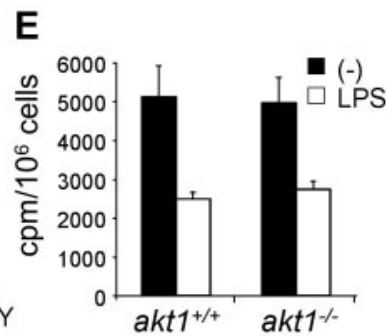

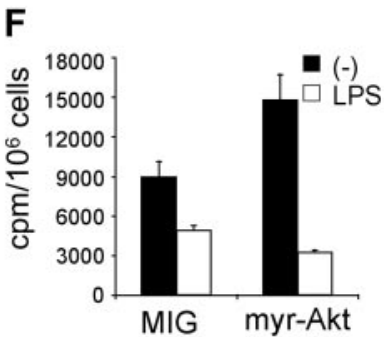

bioenergetic demands of other biologic processes initiated by TLR ligation. To explore this issue, we asked whether glucose metabolism is essential for the maintenance of DC viability after activation by TLRs. This seemed feasible, based on a previous report that DC viability is linked to growth factor-dependent activation of Akt signaling ${ }^{26}$ coupled with our data showing that Akt regulates glycolytic metabolism in DCs (Figure 2). To address this question DC viability after stimulation with LPS was examined under conditions of glucose limitation. DCs activated by various LPS concentrations displayed little cell death under normal glucose conditions (10mM; Figure 3A). However, when stimulated in limited glucose $(2 \mathrm{mM})$, DC viability decreased significantly in an activation-dependent manner (Figure 3A). Analysis over time showed that the increased death of DCs subjected to glucose limitation was probably an acceleration of death normally observed in DC cultures containing $10 \mathrm{mM}$ glucose (Figure 3B), raising the possibility that the DCs were dying after the consumption of available glucose. To test this, DCs were activated in medium containing $10 \mathrm{mM}$ glucose, and on day 2 after stimulation the cultures were fed with media containing either $10 \mathrm{mM}$ or $0 \mathrm{mM}$ glucose, or the existing cultures were supplemented with a bolus of glucose (10mM final concentration). Feeding DCs with glucosesufficient media or supplementation with glucose alone was sufficient to maintain DC viability, whereas DC cultures fed with glucose-free medium underwent apoptosis (Figure 3C). Together the data indicate that glucose is an essential nutrient required for the survival of activated DCs.

Activated DCs produce cytokines that initiate inflammation and help tailor ensuing T-cell responses and express costimulatory molecules that promote interaction with, and activation of, $\mathrm{T}$ cells. The requirement for glucose metabolism to enact these processes was explored by examining DC activation under reduced glucose availability. TLR ligand-induced production of $\mathrm{p} 40$, the common subunit of the inflammatory cytokines IL-12 and IL-23, was significantly reduced in the absence of glucose (Figure 3D); the same was true for tumor necrosis factor- $\alpha$ (supplemental Figure 2). To exclude the possibility that differences in cytokine production in the absence of glucose was the direct consequence of diminished cell numbers due to cell death, DCs were examined for intracellular cytokine expression 5 hours after stimulation, at which time there is little difference in viability between cells cultured with or without glucose (data not shown). In the absence of glucose, LPSstimulated DCs showed a profound defect in the production of $\mathrm{p} 40$ and the up-regulation of the costimulatory molecule CD86, showing that glucose metabolism is essential for DC function (supplemental Figure 3). Likewise, reduced glucose negatively effected baseline expression of CD40 and the ability to up-regulate surface expression of this molecule in response to LPS or PA (supplemental Figure 4). To assess whether inhibition of glycolytic metabolism could affect DC function even when glucose is freely available, DCs were stimulated in the presence of the glycolytic inhibitor 2-deoxyglucose (2-DG). 2-DG profoundly blocked the TLRdependent expression of CD40 (Figure 3E), as well as of CD86 and major histocompatibility complex II (supplemental Figure 5) in a dose-dependent fashion.

To assess the functionality of DCs activated under conditions in which glycolysis is inhibited, the ability of these cells to induce T-cell responses in vivo was measured. DCs were pulsed with OVA alone, or with OVA plus LPS, or OVA plus LPS in medium containing 2-DG for 6 hours, then washed, and injected into the footpads of mice into which CFSE-labeled DO11.10 CD4 T cells had been transferred the day previously. Three days later, draining popliteal LNs were analyzed by flow cytometry to assess the proliferative response of DO11.10 cells. As expected, DCs that had not been pulsed with OVA failed to activate these cells (Figure 3F top). Consistent with their mature phenotype, and the known adjuvant effects of LPS, DCs pulsed with OVA plus LPS induced a markedly stronger T-cell response than did DCs pulsed with OVA alone as measured by CFSE dye dilution of proliferating DO11.10 T cells (Figure 3F). Inclusion of 2-DG during incubation of DCs with OVA plus LPS negated the effects of LPS; T-cell responses induced by these cells were no different from those induced by DCs incubated with OVA alone (Figure 3F).

With the use of CFSE-labeled DCs we tracked whether cells that had been activated under conditions in which glycolysis was inhibited were capable of migrating from the site of injection to the LN and surviving over 2 days. As anticipated, more LPS-activated, OVA-pulsed DCs were present in the LN than was the case for DCs pulsed with OVA in the absence of LPS (Figure 3F bottom). 2-DG treatment had an inhibitory effect on numbers of LPS-activated OVA-pulsed DCs that were recoverable in the draining LN but did not completely negate the effects of LPS in this regard (Figure 3F). 
A

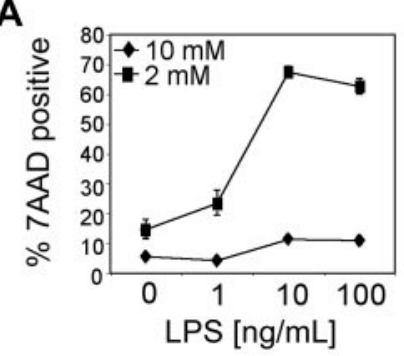

B

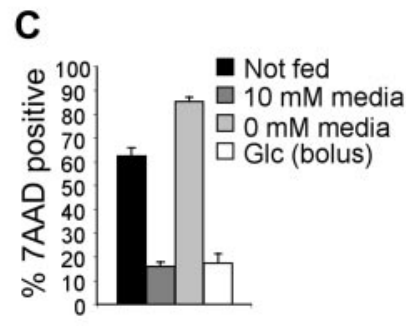

D

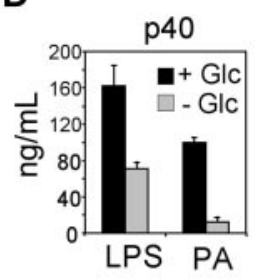

$\mathbf{E}$

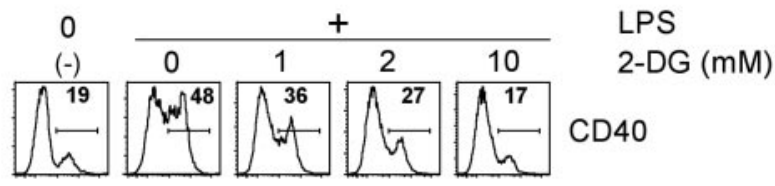

$\mathbf{F}$
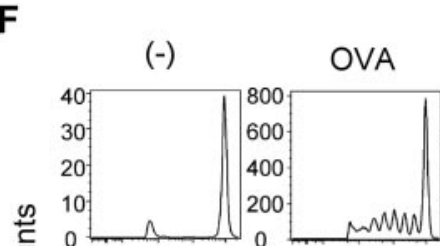

ปั

$$
\begin{array}{r}
\text { ல } 30 \\
20 \\
10 \\
0
\end{array}
$$

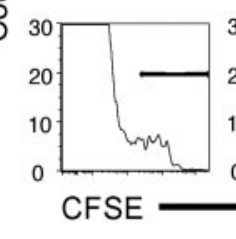

Taken together, these data suggest that initiating glycolytic metabolism at the time of DC activation is important for full DC maturation and subsequent survival and suggest that this metabolic switch broadly underpins the functional changes induced by TLR ligation in DCs required to initiate adaptive immunity.

\section{AMPK antagonizes DC activation}

Our data indicate that resting DCs predominantly use a catabolic program of lipid $\beta$-oxidation/OXPHOS for energy generation and that this pathway is down-regulated after TLR-mediated activation (Figure 1). AMPK is a central regulator of catabolic metabolism and OXPHOS in eukaryotic cells. ${ }^{13}$ To assess whether the increased rates of OXPHOS in resting DCs correlate with AMPK activity we measured the level of AMPK activation after TLR stimulation. AMPK is hyperphosphorylated on Thr-172 in its activation loop (Figure 4A) in resting DCs, and AMPK phosphorylation diminished rapidly after exposure to LPS (Figure 4A). The rapid suppression of AMPK signaling during DC activation led us to hypothesize that AMPK may function to antagonize DC activation. To assess this possibility, AMPK expression was suppressed in DCs through introduction of shRNAs targeting both the $\alpha 1$ and $\alpha 2$ subunits of AMPK by retroviral-mediated gene transfer (supplemental Figure 6). Reduction of AMPK levels promoted an increase in
Figure 3. Glycolysis is essential for cellular activation and survival after exposure to TLR agonists. (A) Survival of DCs 2 days after stimulation with LPS in $10 \mathrm{mM}$ or $2 \mathrm{mM}$ glucose. (B) Survival of DCs over time after stimulation with LPS in media containing either $10 \mathrm{mM}$ or $2 \mathrm{mM}$ glucose. Data points represent the percentage of 7-amino-actinomycin $D(7 A A D)$-positive cells as determined by flow cytometry. (C) Survival of DCs after glucose supplementation. DCs were cultured for 2 days in the presence of $10 \mathrm{mM}$ glucose. On day 2 , the media was either left unchanged (not fed) or supplemented with $10 \mathrm{mM}$ glucose (bolus) or replaced with media containing 10 or OmM glucose. Cell viability was determined 24 hours later by 7AAD uptake. (A-C) Error bars are SDs from triplicate measurements. (D) Cytokine ( $\mathrm{p} 40$ ) production by DCs in response to TLR agonists in the presence or absence of glucose. Cytokine production was determined by enzyme-linked immunoabsorbent assay 18 hours after activation. (E) LPSinduced activation of DCs in the presence of the glycolytic inhibitor 2-DG. Flow cytometry was used to measure surface expression of CD40 after LPS stimulation in the presence of the indicated concentrations of 2-DG. Unstimulated DCs cultured in the absence of 2-DG or LPS are shown for comparison (-). Numbers represent the percentage of cells staining positive for CD40. (F top) DO11.10 CD4 ${ }^{+} \mathrm{T}$-cell proliferative responses, measured by CFSE-dilution, in adoptively transferred mice immunized 4 days previously with DCs alone (-), or DCs pulsed with OVA alone, DCs pulsed with OVA plus LPS, or DCs pulsed with OVA plus LPS in the presence of $10 \mathrm{mM} 2-\mathrm{DG}$. Plots are gated on $\mathrm{KJ} 126{ }^{+} \mathrm{CD}^{+}{ }^{+}$cells. (F bottom) Numbers of CFSE-stained CD11 ${ }^{+}$ cells in LNs draining sites of injection 2 days previously with CFSE-labeled DCs pulsed with OVA alone, pulsed with OVA plus LPS, or pulsed with OVA plus LPS in the presence of $10 \mathrm{mM} 2-\mathrm{DG}$. The panel to the right shows the absence of CFSE signal in control popliteal LNs draining noninjected sites. Bars indicate CFSEpositive cells. All data are representative of 2 to 5 independent experiments.
LPS-induced DC activation as measured by expression of IL12p40 and CD86 (Figure 4B). Activation of AMPK in DCs through the administration of AICAR, a small molecule activator of AMPK, inhibited LPS-mediated IL-12p40 expression (Figure 4C). Moreover, AICAR-treated DCs displayed decreased rates of LPSstimulated glucose consumption. AICAR was as effective as 2-DG, a glycolytic inhibitor, in inhibiting LPS-induced glucose consumption by DCs (Figure 4D). Together these data suggest that AMPK, a known inducer of catabolic metabolism, antagonizes TLR-induced DC maturation and does so, in part, by inhibiting the metabolic switch to glycolysis triggered by TLR stimulation.

\section{IL-10, an inhibitor of DC activation, blocks the switch to glycolytic metabolism}

Consistent with their central role in the initiation of potentially dangerous innate and adaptive responses, DCs and their activation are targeted for regulation by both immune system mediators and pathogen-derived molecules. IL-10 is a potent inhibitor of DC activation made by the immune system. ${ }^{14}$ The similarity between the effects of IL-10 and the inhibition of the switch to glycolytic metabolism on TLR-driven DC activation suggested that the two might be linked. To assess this possibility, LPS-induced glycolysis was measured in DCs cultured with recombinant IL-10 or in the 
A

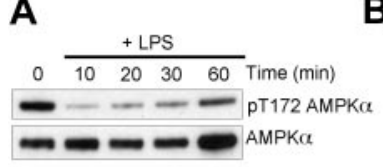

C

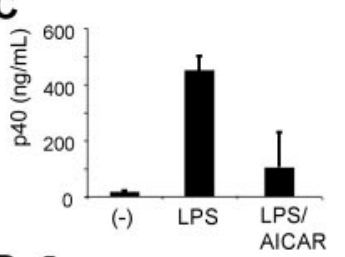

D

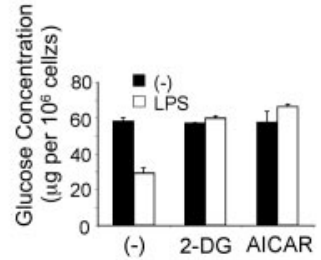

B
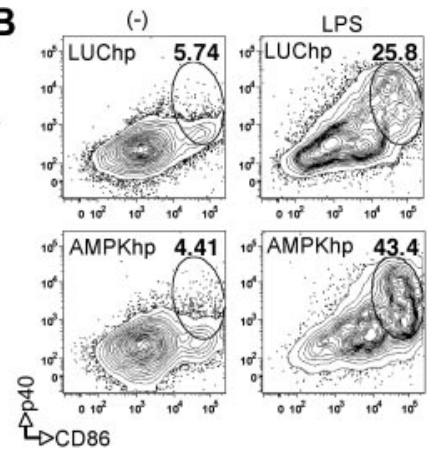

Figure 4. AMPK antagonizes DC activation. (A) Extracts of DCs stimulated with LPS for the times shown were probed by Western blotting with antibodies specific for phosphorylated Thr-172 AMPK $\alpha$ or for total AMPK $\alpha$. (B) LPS-induced maturation, measured as $\mathrm{p} 40$ production and CD86 expression, as measured by flow cytometry in DCs transduced with AMPK shRNAs or control Luc shRNAs. (-) denotes resting DCs; numbers indicate percentage of $\mathrm{CD}_{11 \mathrm{c}^{+}}$cells within gates. (C) p40 production by resting DCs (-) and DCs stimulated with LPS or LPS plus AICAR. Cytokine production was determined by enzyme-linked immunoabsorbent assay 18 hours after activation. (D) Glucose consumption (measured as glucose remaining in culture medium after 24 hours of culture, normalized per $10^{6}$ cells) by DCs cultured without or with LPS in the absence (-) or presence of 2-DG or AICAR. Bars represent mean values \pm SDs from 3 independent replicates. All data are representative of 2 or more independent experiments.

presence of an $\mathrm{Ab}$ against the IL-10R, which blocks the effects of endogenous IL-10 made by TLR ligand-activated DCs. ${ }^{27}$ TLR-initiated glycolytic metabolism was inhibited in the presence of IL-10 and increased when IL-10 feedback was blocked (Figure 5A), suggesting that IL-10 negatively regulates glycolytic metabolism in DCs.

IL-10 has been reported to inhibit LPS-induced Akt phosphorylation, ${ }^{9}$ suggesting one possible mechanism by which IL-10 may block TLR-induced glycolysis. However, we were unable to detect a measurable effect of IL-10 on AKT phosphorylation (data not shown). Because AMPK activation can block TLR-induced stimulation of glycolytic metabolism in DCs, and AMPK activation is negatively regulated by LPS, we assessed the effects of IL-10 signaling on AMPK activation. IL-10 by itself did not display a profound effect on AMPK activation but was found to antagonize the LPS-induced hypophosphorylation of AMPK (Figure 5B).

The observation that IL-10 can inhibit TLR-mediated glycolysis in DCs raises the possibility that the inhibitory effects of 2-DG and AICAR on DC maturation (Figures 3-4) may simply be the result of autocrine IL-10 production by DCs. To address this issue, we examined the effects of these small molecules on LPS-induced DC activation with the use of DCs derived from IL- $10^{-1-}$ mice. The activation of IL-10-deficient DCs, as measured by LPS-stimulated CD40 expression, was profoundly inhibited by 2-DG and AICAR (Figure 5C). Similarly, the production of p40 (Figure 5D) and tumor necrosis factor- $\alpha$ (supplemental Figure 7) was inhibited by 2-DG in IL-10 ${ }^{-1-}$ DCs, suggesting that the effect of glycolytic inhibition on DC activation is IL-10 independent. Interestingly, IL-10 production by wild-type DCs in response to LPS was also suppressed by 2-DG and AICAR (supplemental Figure 8). Together, these data suggest that DCs respond to IL-10 by retaining a metabolic profile that is incompatible with enactment of the activation pathway.
A

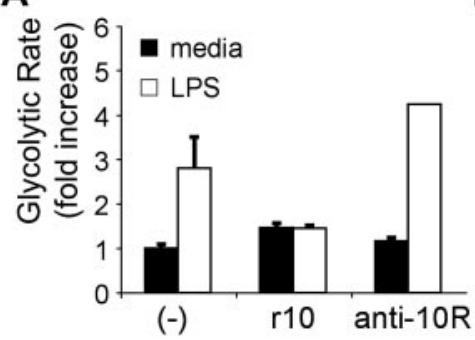

Figure 5. IL-10 inhibits the metabolic switch to glycolysis induced by TLR agonists. (A) Fold increase in the glycolytic rate of DCs cultured without or with LPS in the absence (-) or presence of recombinant IL-10 (r10) or anti-IL-10R Ab (anti-10R). (B) AMPK phosphorylation in DCs stimulated with LPS, IL-10, or LPS plus IL-10. DCs were treated as indicated for 30 minutes, and lysates were probed by Western blotting with antibodies specific for phosphorylated AMPK $\alpha$ (Thr-172), or for total AMPK $\alpha$ or for actin (as loading controls). (C) LPS-induced expression of CD40 by IL-10 $10^{-1-}$ DCs is inhibited by exogenous IL-10, 2-DG, and AICAR. CD40 expression at 6 hours after stimulation was measured by flow cytometry. (-) denotes unstimulated DCs. Plain numbers represent the percentage of cells within the positive gate, and bold italic numbers represent mean fluorescence intensities. (D) IL-10 and 2-DG are equally potent at inhibiting LPS-induced cytokine ( $\mathrm{p} 40$ ) production. Cytokine levels in supernatants from cells cultured for 18 hours without (-) or with LPS, plus minus IL-10 or 2-DG, were measured by enzyme-linked immunoabsorbent assay. Bars represent mean values \pm SDs from 3 independent replicates. All data are representative of 2 or more independent experiments. (E) A summary of findings.

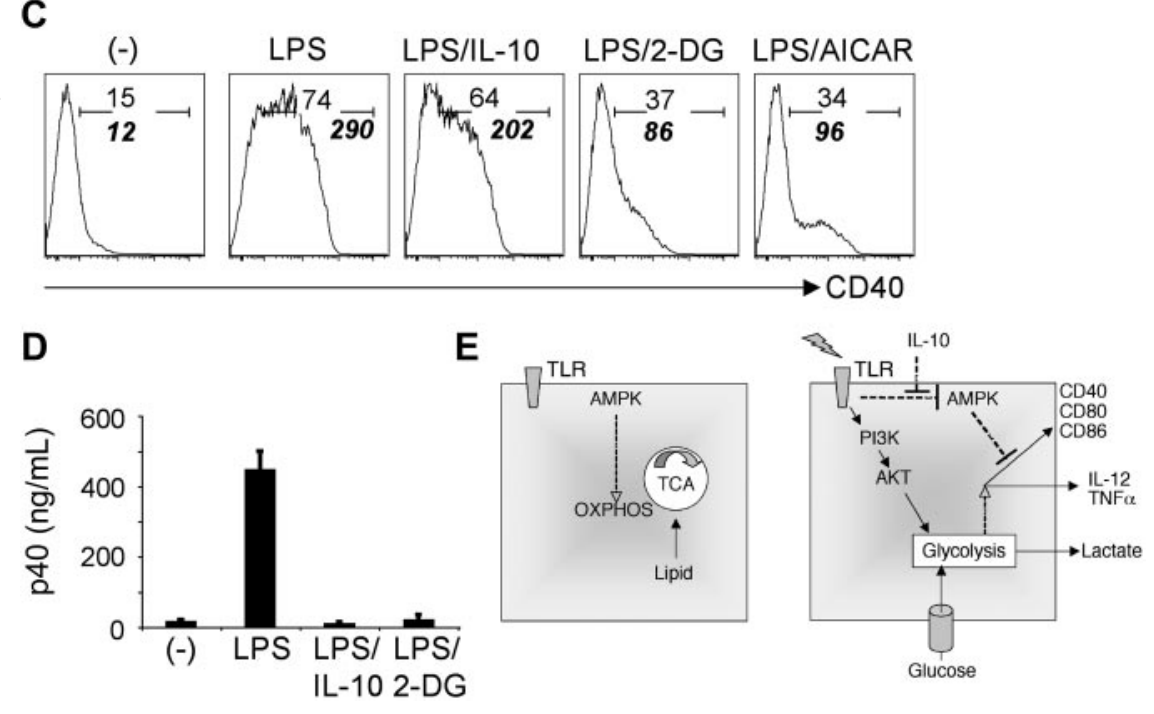




\section{Discussion}

DCs play a central role in the induction of host-defense mechanisms. Of critical importance is their ability to rapidly switch from a resting state to an activated state in response to environmental danger signals associated with infection or cellular damage. We report here that TLR signaling, a major pathway for DC activation, triggers metabolic reprogramming that is essential for full DC maturation and associated biologic functions. This metabolic conversion is characterized by a switch from mitochondrial OXPHOS fueled by the $\beta$-oxidation of lipids to aerobic glycolysis. The TLR-stimulated change in DC metabolism, which is similar to the Warburg metabolism observed in transformed cells, is driven by the PI3K/Akt pathway and is antagonized by AMPK. The immunosuppressive cytokine IL-10, which inhibits TLR-stimulated DC activation, was found to partially antagonize the LPS-induced hypophosphorylation of AMPK and, thus, inhibits TLR-stimulated glycolysis in DCs. Together our data suggest that the PI3K-Akt and AMPK pathways function in opposing fashions to regulate TLRstimulated metabolism and DC activation (Figure 5E). In the absence of TLR signals, activated AMPK, by favoring OXPHOS over glycolysis, functions to enforce a metabolic profile incompatible with DC activation. TLR signaling promotes a switch to glycolytic metabolism by activating PI3K-Akt signaling and antagonizing AMPK signaling. IL-10 may function to inhibit glycolysis and DC activation, in part, by preventing TLR-mediated AMPK hypophosphorylation. Together our data highlight the importance of TLR-dependent regulation of metabolism for proper DC maturation and function.

Why do DCs initiate such a dramatic change in their core metabolism in response to TLR signals? From the standpoint of bioenergetics, glycolysis is capable of producing ATP less efficiently but more quickly than OXPHOS. Engaging in increased glycolytic metabolism may function as a protective mechanism to preserve cellular ATP levels and maintain DC viability during an immune response. Moreover, the ability to generate ATP anaerobically would be advantageous in infection/inflammation settings where oxygen tension can be low. Consistent with this hypoxia inducible factor- $1 \alpha$ has been reported to enhance LPS-induced glycolytic activity in DCs. ${ }^{28}$ The metabolism of glucose to pyruvate and other metabolites serves the additional function of generating intermediates for de novo synthesis of amino acids and lipids. ${ }^{25,29}$ In contrast to the situation in $\mathrm{T}$ cells and cancer cells, where the switch to Warburg metabolism is believed to primarily fuel cell division, ${ }^{25,30}$ the Warburg effect in DCs is not linked to cellular proliferation but rather appears to support the development of demanding new cellular functions and biosynthetic activities, including the synthesis and secretion of a broad panel of immune mediators.

By switching their cellular bioenergetics in favor of glycolytic metabolism, TLR-activated DCs are increasingly reliant on glucose for survival and function and become more sensitive to death by nutrient limitation. This raises the possibility that the signal transduction pathways that regulate bioenergetics play a central role not only in early events associated with DC activation but additionally in the regulation of DC lifespan; such a concept is consistent with a previous report that Akt is critical for DC survival after proinflammatory signaling. ${ }^{26}$ It is important to stress that in the absence of activation signals, DCs are able to survive adequately in low-glucose conditions, presumably because they are generating energy through alternate means (ie, $\beta$-oxidation of lipids) and have relatively low biosynthetic demands.

Our data strongly implicate the PI3K/Akt pathway in the TLR-induced regulation of DC metabolism. Previous reports have shown that TLRs activate PI3K in a myeloid differentiation primary response gene 88 -dependent manner. ${ }^{31}$ Akt is a major downstream target of $\mathrm{PI}_{3} \mathrm{~K}^{23}$ and plays a multifaceted role in promoting glycolysis by stimulating surface membrane expression of glucose transporter 1, which by increasing the cytoplasmic concentration of glucose favors increased glycolysis, ${ }^{24}$ and by activating hexokinase and phosphofructokinase, which are ratelimiting enzymes in glycolysis. ${ }^{25}$ Growth factor receptors such as that for GM-CSF also activate PI3K/Akt, and this may account for the background levels of active Akt we observed in immature resting DCs, which are grown in this cytokine. ${ }^{15}$ However, TLR ligation clearly provides an additional and decisive stimulus for Akt activation that leads to an increase in the glycolytic rate, which is Akt dependent. Our data indicate that this aspect of TLR signaling effectively provides metabolic permission for the enactment of the TLR-initiated signals for full DC activation transmitted by the mitogen-activated protein kinase and nuclear factor- $\kappa \mathrm{B}$ pathways. ${ }^{32}$

Our data implicate AMPK as an inhibitor of DC activation. AMPK is activated by at least 3 physiologically activated pathways: (1) increases in the AMP/ATP ratio, (2) increases in intracellular $\mathrm{Ca}^{2+}$, and (3) cytokine signaling mediated by the transforming growth factor- $\beta$-associated kinase- $1 .{ }^{33,34}$ Among other functions, AMPK stimulates metabolic pathways that generate ATP, including fatty acid oxidation and autophagy. ${ }^{33-35}$ AMPK activation in immature DCs may enforce a metabolic program of quiescence that relies primarily on OXPHOS for ATP generation. By shifting metabolic activity away from glycolysis, AMPK activation appears to establish a bioenergetic status quo in DCs that is incompatible with cellular maturation. The rapid downregulation of AMPK by TLR signaling, coupled with increased PI3K/Akt-dependent glycolysis, may be a key step in switching the bioenergetic state of DCs to one that supports full maturation and activation. The downstream effects of AMPK activation are complex, and further work is required to understand how AMPK regulates TLR-induced DC activation. This is particularly important, given that AMPK is a target of a number of widely prescribed drugs, including metformin, used for the treatment of diabetes. ${ }^{33,34}$ Recent evidence suggests that metformin can alter the development of CD8 memory T cells, possibly through the action of AMPK. ${ }^{36}$ This raises the possibility that drugs approved for metabolic disorders may have striking implications for immunity and may be useful for the control of immunologic disorders.

DC activation is targeted by both immune system mediators and pathogen-derived molecules to control the initiation and extent of the immune response. Our findings suggest that antiinflammatory mediators, such as IL-10, may function in part by limiting the glycolytic switch in DCs that accompanies activation. IL-10 plays a central role in preventing immunopathology during innate and adaptive immune responses to pathogens and is a key effector cytokine of regulatory $\mathrm{T}$ cells, which are crucial for selftolerance. ${ }^{37,38}$ Our data show that IL-10 antagonizes TLR-induced glycolysis that is driven by PI3K/Akt signaling in DCs. It is tempting to believe that this reflects an effect of IL-10 on the activation of AMPK pathway, because in our studies IL-10 partially antagonizes LPS-stimulated AMPK hypophosphorylation. Of interest in this context is that AMPK is activated by IL- 6 and leptin, ${ }^{13}$ cytokines which, like IL-10, signal through a STAT3-dependent 
pathway. ${ }^{39}$ Future efforts will focus on the relationships between these cytokines and their effects on metabolic transitions in DCs.

By switching toward a program of glycolytic metabolism, TLR-activated DCs are increasingly reliant on glucose for survival and function, and they become more sensitive to death by nutrient limitation. This raises the possibility that the signal transduction pathways that regulate bioenergetics play a central role not only in early events associated with DC activation but additionally in the regulation of DC lifespan. This view may explain how IL-10, an inhibitory cytokine shown here to block the TLR-induced switch to glycolysis, can have such broad effects on the expression of innate inflammatory responses and adaptive immunity. ${ }^{40}$ Our data suggest that the manipulation of metabolic pathways in DCs may provide mechanisms for broadly inhibiting inflammatory processes and pathologic cellular function.

\section{Acknowledgments}

We thank Lucas Carvalho, Aleah Roberts, and Erika Pearce for helpful discussions.

This work was supported by the Human Frontiers Scientific Program (C.M.K.), the Cancer Research Institute and the Canadian
Institutes for Health Research (MOP-93 799; R.G.J.), and the National Institutes of Health (T32 training award AI049823, E.A.; CA104838, C.B.T.; AI53825, E.J.P.).

\section{Authorship}

Contribution: C.M.K., J.S., and R.G.J. designed the research, performed experiments, and interpreted data; T.H., J.B., E.A., R.J.D., J.R.C., and E.J.P. performed experiments; T.H., J.B., E.A., R.J.D., J.R.C., and C.B.T. assisted with experimental design and data interpretation; C.M.K., R.G.J., and E.J.P. wrote the paper; and E.J.P. designed and directed the research and interpreted data.

Conflict-of-interest disclosure: The authors declare no competing financial interests.

The current affiliation for C.M.K. is Merck Research Laboratories, Department of Biochemistry and Molecular Biology, Merck Frosst Canada \& Co Inc, Kirkland, QC. The current affiliation for J.S. is Beirne B. Carter Center for Immunology Research, University of Virginia, Charlottesville, VA.

Correspondence: Edward J. Pearce, Trudeau Institute, 154 Algonquin Ave, Saranac Lake, NY 12983; e-mail: ejpearce@ trudeauinstitute.org.

\section{References}

1. Barton GM, Medzhitov R. Control of adaptive immune responses by Toll-like receptors. Curr Opin Immunol. 2002;14(3):380-383.

2. Krawczyk CM, Shen $\mathrm{H}$, Pearce EJ. Functional plasticity in memory $T$ helper cell responses. J Immunol. 2007;178(7):4080-4088.

3. Akira S, Takeda K. Toll-like receptor signalling. Nat Rev Immunol. 2004;4(7):499-511.

4. Iwasaki A, Medzhitov R. Toll-like receptor control of the adaptive immune responses. Nat Immunol. 2004;5(10):987-995.

5. Huang $Q$, Liu D, Majewski $P$, et al. The plasticity of dendritic cell responses to pathogens and their components. Science. 2001;294(5543):870-875.

6. Angeli V, Randolph GJ. Inflammation, lymphatic function, and dendritic cell migration. Lymphat Res Biol. 2006;4(4):217-228.

7. Shaw RJ. Glucose metabolism and cancer. Curr Opin Cell Biol. 2006;18(6):598-608.

8. Christofk HR, Vander Heiden MG, Harris MH, et al. The M2 splice isoform of pyruvate kinase is important for cancer metabolism and tumour growth. Nature. 2008;452(7184):230-233.

9. Bhattacharyya S, Sen P, Wallet M, Long B, Baldwin AS Jr, Tisch R. Immunoregulation of dendritic cells by IL-10 is mediated through suppression of the PI3K/Akt pathway and of IkappaB kinase activity. Blood. 2004;104(4):1100-1109.

10. Ohtani M, Nagai S, Kondo S, et al. Mammalian target of rapamycin and glycogen synthase kinase 3 differentially regulate lipopolysaccharideinduced interleukin-12 production in dendritic cells. Blood. 2008;112(3):635-643.

11. Xu Y, Jagannath $C$, Liu XD, Sharafkhaneh $A$, Kolodziejska KE, Eissa NT. Toll-like receptor 4 is a sensor for autophagy associated with innate immunity. Immunity. 2007;27(1):135-144.

12. Sanjuan MA, Dillon CP, Tait SW, et al. Toll-like receptor signalling in macrophages links the autophagy pathway to phagocytosis. Nature. 2007; 450(7173):1253-1257.

13. Hardie DG. AMP-activated/SNF1 protein kinases: conserved guardians of cellular energy. Nat Rev Mol Cell Biol. 2007;8(10):774-785.

14. Murray PJ. Understanding and exploiting the endogenous interleukin-10/STAT3-mediated anti- inflammatory response. Curr Opin Pharmacol. 2006;6(4):379-386.

15. Lutz MB, Kukutsch N, Ogilvie AL, et al. An advanced culture method for generating large quantities of highly pure dendritic cells from mouse bone marrow. J Immunol Methods. 1999;223(1):77-92.

16. Sun J, Krawczyk CJ, Pearce EJ. Suppression of Th2 cell development by Notch ligands Delta1 and Delta4. J Immunol. 2008;180(3):1655-1661.

17. Jones RG, Bui T, White $\mathrm{C}$, et al. The proapoptotic factors Bax and Bak regulate $T$ cell proliferation through control of endoplasmic reticulum $\mathrm{Ca}(2+)$ homeostasis. Immunity. 2007;27(2):268-280.

18. MacDonald AS, Straw AD, Bauman B, Pearce EJ. CD8- dendritic cell activation status plays an integral role in influencing Th2 response development. J Immunol. 2001;167(4):1982-1988.

19. Elstrom RL, Bauer DE, Buzzai M, et al. Akt stimulates aerobic glycolysis in cancer cells. Cancer Res. 2004;64(11):3892-3899.

20. Deberardinis RJ, Lum JJ, Thompson CB. Phosphatidylinositol 3-kinase-dependent modulation of carnitine palmitoyltransferase $1 \mathrm{~A}$ expression regulates lipid metabolism during hematopoietic cell growth. J Biol Chem. 2006;281(49):37372-37380.

21. Lum JJ, Bui T, Gruber M, et al. The transcription factor HIF-1alpha plays a critical role in the growth factor-dependent regulation of both aerobic and anaerobic glycolysis. Genes Dev. 2007;21(9):1037-1049.

22. Fox CJ, Hammerman PS, Thompson CB. Fuel feeds function: energy metabolism and the T-cell response. Nat Rev Immunol. 2005;5(11):844-852.

23. Engelman JA, Luo J, Cantley LC. The evolution of phosphatidylinositol 3-kinases as regulators of growth and metabolism. Nat Rev Genet. 2006; 7(8):606-619.

24. Rathmell JC, Elstrom RL, Cinalli RM, Thompson $\mathrm{CB}$. Activated Akt promotes increased resting $\mathrm{T}$ cell size, CD28-independent $\mathrm{T}$ cell growth, and development of autoimmunity and lymphoma. Eur J Immunol. 2003;33(8):2223-2232.

25. Jones RG, Thompson CB. Revving the engine: signal transduction fuels $\mathrm{T}$ cell activation. Immunity. 2007;27(2):173-178.

26. Park D, Lapteva N, Seethammagari M, Slawin $\mathrm{KM}$, Spencer DM. An essential role for Akt1 in dendritic cell function and tumor immunotherapy. Nat Biotechnol. 2006;24(12):1581-1590.
27. Kane CM, Cervi L, Sun J, et al. Helminth antigens modulate TLR-initiated dendritic cell activation. J Immunol. 2004;173(12):7454-7461.

28. Jantsch J, Chakravortty D, Turza N, et al. Hypoxia and hypoxia-inducible factor-1 alpha modulate lipopolysaccharide-induced dendritic cell activation and function. J Immunol. 2008;180(7): 4697-4705.

29. Christofk HR, Vander Heiden MG, Wu N, Asara $\mathrm{JM}$, Cantley LC. Pyruvate kinase M2 is a phosphotyrosine-binding protein. Nature. 2008; 452(7184):181-186.

30. Frauwirth KA, Riley JL, Harris $\mathrm{MH}$, et al. The CD28 signaling pathway regulates glucose metabolism. Immunity. 2002;16(6):769-777.

31. Gelman AE, LaRosa DF, Zhang J, et al. The adaptor molecule MyD88 activates PI-3 kinase signaling in CD4 + T cells and enables CpG oligodeoxynucleotide-mediated costimulation. Immunity. 2006;25(5):783-793.

32. Kawai T, Akira S. TLR signaling. Semin Immunol. 2007;19(1):24-32.

33. Hardie DG. AMPK and SNF1: snuffing out stress. Cell Metab. 2007;6(5):339-340.

34. Hardie DG. AMPK: a key regulator of energy balance in the single cell and the whole organism. Int J Obes (Lond). 2008;32(suppl 4):S7-S12.

35. Poels J, Spasic MR, Callaerts P, Norga KK. Expanding roles for AMP-activated protein kinase in neuronal survival and autophagy. BioEssays. 2009;31(9):944-952.

36. Pearce EL, Walsh MC, Cejas PJ, et al. Enhancing CD8 T-cell memory by modulating fatty acid metabolism. Nature. 2009;460(7251):103-107.

37. Moore KW, de Waal Malefyt R, Coffman RL, O'Garra A. Interleukin-10 and the interleukin-10 receptor. Annu Rev Immunol. 2001;19:683-765.

38. O'Garra A, Vieira P. T(H)1 cells control themselves by producing interleukin-10. Nat Rev Immunol. 2007;7(6):425-428.

39. El Kasmi KC, Holst J, Coffre M, et al. General nature of the STAT3-activated anti-inflammatory response. J Immunol. 2006;177(11):7880-7888.

40. O'Shea JJ, Murray PJ. Cytokine signaling modules in inflammatory responses. Immunity. 2008; 28(4):477-487. 